\title{
New call blocking probability (NCBP) in improved adaptive quality of service (AdQoS) for wireless multimedia communications using hierarchical cellular approach
}

\begin{abstract}
The maintenance of quality of service (QoS) in a wireless environment is a difficult problem. Most researchers have already acknowledged adaptive techniques as the appropriate method to deal with the fluctuation of QoS in a wireless cellular network. This paper proposes an improved new call blocking probability (NCBP) from an adaptive QoS (AdQoS) to support the QoS guarantee. Besides using an adaptive technique, this method utilizes a hierarchical cellular approach with a bandwidth reallocation algorithm to determine the acceptance or rejection of a new call. The approach would improve the NCBP performance of the multimedia application over high-speed wireless cellular networks.
\end{abstract}

Keyword: New call blocking probability (NCBP); Adaptive quality of service (AdQoS); Wireless communication; Quality of service 